\title{
Calendar of Events
}

$6^{\text {th }}$ World Congress of Neurorehabilitation

March 21-25, 2010

Vienna, Austria

Website www.wcnr2010.org

American Academy of Neurology

$62^{\text {nd }}$ Annual Meeting

April 10-17, 2010

Toronto, Ontario, Canada

Website http://am.aan.com/

\section{$\bullet$}

American Occupational Therapy Association

$90^{\text {th }}$ Annual Conference and Expo

April 29-May 2, 2010

Orlando, FL

Website http://www.aota.org/ConfandEvents/

$\underline{\text { 2010AnnualConference.aspx }}$

\section{$\bullet$}

International Child Neurology Association

$11^{\text {th }}$ International Child Neurology Congress

May 2-7, 2010

Cairo, Egypt

Website http://www.icnc2010.com/
The Pediatric Orthopedic Society of North America

$$
\text { May 3-7, } 2010
$$

Waikaloa, HI

Website http://www.posna.org/

European Society of Movement Analysis for Adults and Children (ESMAC)

$19^{\text {th }}$ Annual General Meeting

\section{May 10-14, 2010}

Miami, FL

Website Website http://www.esmac.org/

$2^{\text {nd }}$ Joint Meeting of European Society of Movement Analysis for Adults and Children \& Gait and Clinical Movement Analysis Society

\section{May 12-15, 2010}

Miami, FL

Website http://www.gcmas.org/conference

$22^{\text {nd }}$ Annual Meeting of the European Academy of Childhood Disability

\section{May 27-29, 2010}

Brussels, Belgium

Website http://www.eacd2010.com 
American Urological Association

May 29-June 3, 2010

San Francisco, CA

Website http://www.auanet.org

Association of Children's Prosthetic-Orthotic Clinics 2010 Annual Meeting

June 2-5, 2010

Clearwater, FL

Website http://www.acpoc.org/meeting/

American Physical Therapy Association Annual Conference

June 16-19, 2010

Boston, MA

Website http://www.apta.org

Society for Research into Hydrocephalus and Spina Bifida

$54^{\text {th }}$ Scientific Meeting

July 7-10, 2010

University of British Columbia

Vancouver, Canada

Website http://srhsb.org/

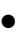

The American Academy of Cerebral Palsy and Developmental Medicine

September 22-25, 2010

Marriott Wardman Park Hotal

Washington DC, USA

Website: http://www.aacpdm.org/meeting/2010
American Academy of Pediatrics

AAP National Conference and Exhibition (NCE)

October 2-5, 2010

San Francisco, CA

Website: http://www.aapexperience.org/

International Child Neurology Association \& International Cerebral Palsy Society

$8^{\text {th }}$ International Congress on Cerebral Palsy

October 13-16, 2010

Ljubljana, Slovenia

Website http://www.cpljubljana2010.eu

Closing the Gap

$28^{\text {th }}$ Annual Conference

October 21-23, 2010

Bloomington, $\mathrm{MN}$

Website: http://www.closingthegap.com/conference/

$71^{\text {st }}$ Annual Assembly of American Academy of Physical Medicine and Rehabilitation

November 4-7, 2010

Seattle, WA

Website: http://www.aapmr.org

$60^{\text {th }}$ Congress of Neurological Surgeons Annual Meeting

October 16-21, 2010

San Francisco, CA

Website: http://www.neurosurgeon.org/ 